\title{
Chest Wall Abscess with Bone Invasion in Infant after BCG Vaccination
}

\author{
Dong Seok Shin (D), Min Hyub Choi (D), Woo Young Choi (D), Ji Seon Cheon (D), Kyung Min Son (D) \\ Department of Plastic and Reconstructive Surgery, Chosun University College of Medicine, Gwangju, Korea
}

\begin{abstract}
The bacillus Calmette-Guérin (BCG) vaccine is a safe live vaccine mainly administered to infants to prevent tuberculosis; complications rarely occur after administration of the BCG vaccine. Herein, we report the case of an infant who developed a rare complication of a tuberculous abscess in the chest wall and osteomyelitis in the ribs after administration of the BCG vaccine at 1 month of age. An 11-month-old male infant was admitted to the hospital due to a palpable mass in the right anterolateral chest area detected about a month before hospitalization, with no tenderness, erythema, or lymphadenopathy. After thoracic computed tomography and ultrasonography, the cause of the abscess was suspected to be mycobacterial infection and the abscess was removed under general anesthesia. Chronic granulomatous inflammation with caseous necrosis was detected through a biopsy, and polymerase chain reaction was performed and Mycobacterium tuberculosis complex was detected. However, staining for acid-fast bacteria and microbiological cultures were negative. Based on these results, the patient was diagnosed with a tuberculous abscess and osteomyelitis in the chest wall owing to administration of the BCG vaccine. After being medicated with isoniazid and rifampicin for 6 months, the patient was successfully treated.
\end{abstract}

Keywords: Tuberculous abscess; BCG vaccine; Tuberculosis; Infant

\section{Introduction}

The bacillus Calmette-Guérin (BCG) vaccine is a safe live vaccine derived from an attenuated strain of Mycobacterium bovis and mainly administered on infants for prevention of tuberculosis. The rate of complications after inoculation of the BCG vaccine is relatively low; the reported complications include local skin reactions, abscesses, regional lymphadenitis, osteomyelitis, and disseminated infections [1]. The most common complications include formation of a subcutaneous abscess around the inoculation site and regional lymphadenitis [2,3]. Osteomyelitis is a systemic adverse reaction that rarely occurs after administration of the BCG vaccine. Although complications from BCG vaccination generally have a good prognosis, without proper diagnosis and treatment they can lead to severe sequelae [3,4]. We report a case of an 11-month-old male infant who developed a BCG-induced Mycobacterial abscess in the chest wall and osteomyelitis of the rib after administration of the BCG vaccine. The study protocol conformed to the ethical guidelines of the Declaration of Helsinki, and a written informed consent was obtained from the patient's parents in accordance with the Chosun University Hospital Institutional Review Board (IRB No. 2020-03-003).

\section{Case}

An 11-month-old male infant developed a palpable mass approximately $6 \times 3 \mathrm{~cm}$ in size on the right anterolateral chest wall on the right 5 th to 8 th ribs a month before

\section{Case Report}

Received: February 5, 2020

Revised: March 20, 2020

Accepted: March 31, 2020

Corresponding author: Woo Young Choi, M.D.

Department of Plastic and Reconstructive Surgery, Chosun University College of Medicine, 365 Pilmun-daero, Dong-gu, Gwangju 61453, Korea

Tel: +82-62-220-3180

Fax: +82-62-225-0996

E-mail: wychoi@chosun.ac.kr

This is an Open Access article distributed under the terms of the Creative Commons Attribution Non-Commercial License (https://creativecommons.org/licenses/by-nc/4.0/) which permits unrestricted non-commercial use, distribution, and reproduction in any medium, provided the original work is properly cited.

(c) 2020 Korean Wound Management Society 
presentation. The infant had a full-term normal delivery and weighed $3.1 \mathrm{~kg}$ at birth. The medical history of the infant did not include signs of immunodeficiency, since blood tests performed at birth were normal and scheduled vaccinations were administered without significant complications. The patient had a history of BCG vaccination on the left upper arm 10 months earlier; at the inoculation site there were no signs of infection except for the presence of linear scars with mild redness. The patient had no history of chest injury, no contact with tuberculosis patients, and no history of medications.

The lesion was not a sign of infection; there was no tenderness, erythema, or warmth. Moreover, lymphadenopathy was not observed during the physical examination (Fig. 1). Blood tests were performed, and revealed that the erythrocyte sedimentation rate $(14 \mathrm{~mm} / \mathrm{hr})$, white blood cell count $(15,070$ $\left.\mathrm{mm}^{3}\right)$, and C-reactive protein levels $(0.44 \mathrm{mg} / \mathrm{L})$ were normal.

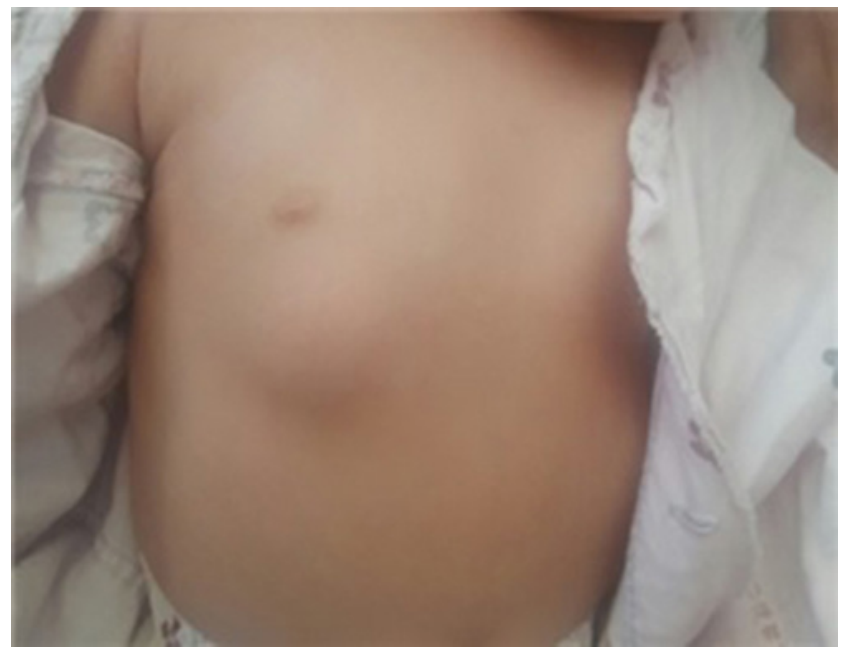

Fig. 1. Preoperative view of protruding mass in the right chest wall.
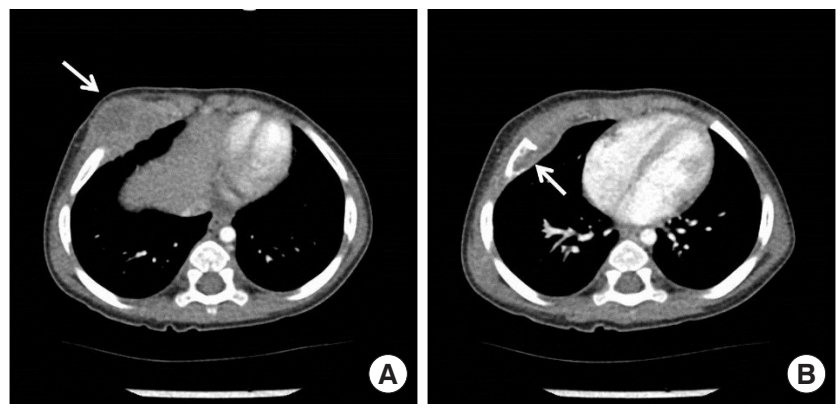

Fig. 2. Preoperative radiological images. (A) Preoperative computed tomography scans. Approximately $7 \times 2.6 \mathrm{~cm}$ multiseptated mass (arrow) on the anterolateral chest. (B) Approximately $1.5 \mathrm{~cm}$ osteolytic bone lesion (arrow) in the right 5th rib.
In addition, fever, weakness, loss of appetite, cough, phlegm, hemoptysis, and weight loss were not observed. Computed tomography and ultrasonography were performed. In the thoracic computed tomography, a clustered or multiseptated low attenuation mass $(7 \times 2.6 \mathrm{~cm})$ with a peripheral rim enhancement was detected on the anterolateral aspect of the right chest wall on the anterior arcs of the right 5th to 8th ribs (Fig. 2A). An osteolytic bone lesion was present on the adjacent anterior arc of the 5th rib (Fig. 2B). The radiologist reported that the cause of the chest wall abscess and adjacent osteomyelitis with moderate chronicity was a mycobacterial infection.

There was no active parenchymal lesion on either of the lungs with definite evidence of active tuberculosis pneumonia. Diagnostic ultrasonography and fine-needle aspiration were performed, indicating no change in the size of the abscess; hence, the presence of a soft tissue abscess rather than a hematoma was confirmed.

Surgery was performed under general anesthesia for reconfirmation and removal of the mass. We found the abscess in the subcutaneous layer. Pus was observed when the dissection took place. Irrigation was performed to remove the pus and devitalized tissues were removed. We then conducted debridement and curettage on the bone erosion area without resecting the bone. A thoracic surgeon checked for the presence of invasions in the thoracic cage and found none. The tissue obtained during surgery was submitted for histopathological examination, where chronic granulomatous inflammation with caseous necrosis was observed. Polymerase chain reaction was conducted for the detection of Mycobacterium tuber-

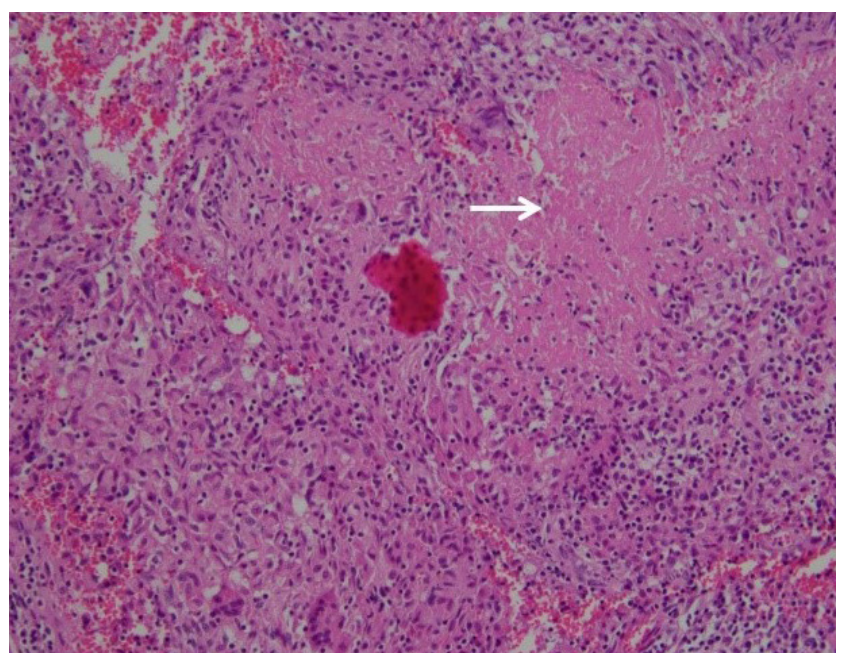

Fig. 3. Biopsy image of the abscess. Chronic granulomatous inflammation with caseous necrosis (arrow) $(H \& E, \times 100)$. 

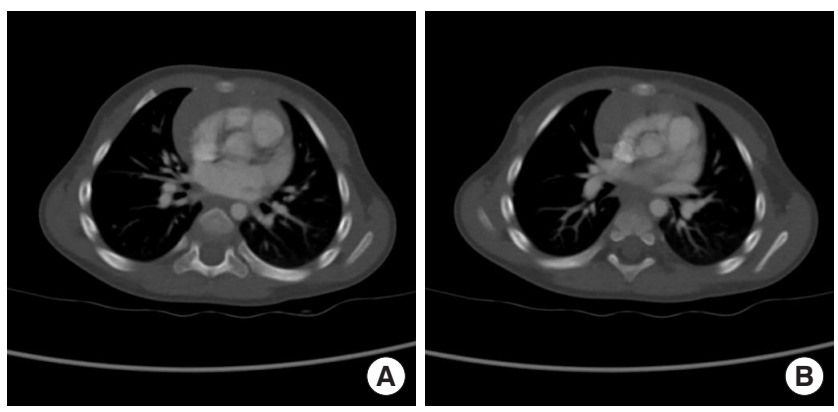

Fig. 4. Computed tomography images of the patient 9 months after surgery. (A) Near complete postoperative improvement in the right anterior chest wall. (B) The osteolytic bone lesion is improved.

culosis complex and the result was positive (Fig. 3). Acid-fast bacteria and microbiological cultures were found to be negative for tuberculosis.

Based on the test results and the patient's medical history, we diagnosed the patient as having tuberculous abscess and osteomyelitis caused by BCG vaccine. After treatment with isoniazid $(10 \mathrm{mg} / \mathrm{kg})$ and rifampicin $(10 \mathrm{mg} / \mathrm{kg})$ for 6 months, the symptoms improved and no complications were observed (Fig. 4).

\section{Discussion}

The BCG vaccine is a live vaccine used worldwide to prevent tuberculosis. In Korea, $96 \%$ to $97 \%$ of infants receive BCG vaccination at 4 weeks after birth [2]. The rate of complications after administration of the BCG vaccine is $0.01 \%$ to $3.6 \%$; the most common complications include formation of abscess around the inoculation site and regional lymphadenitis $[2,5]$. In the present report, no lymphadenitis was observed, but the location of the abscess in the anterior chest instead of at the inoculation site was noted. Though prognoses of complications from administration of the BCG vaccine is good among immunocompetent children, when a rare complication such as osteomyelitis occurs, delayed treatment may lead to impaired growth of the invaded bone, fistula in the surrounding tissues, or recurrence of the abscess [3]. However, in our case, the infant did not show any sign of infection and preoperative radiological examination was the only modality that revealed invasion in the rib. Debridement and curettage were performed without bone resection in the invaded rib area, and the bone lesion showed improvement in the postoperative follow-up computed tomography.

The treatment of complications from administering the
BCG vaccine in the chest wall has been previously discussed $[4,6]$. Since the delayed treatment of osteitis or osteomyelitis from BCG vaccine can lead to irreversible sequelae in childaged patients, surgical removal and anti-tuberculosis drug treatment are recommended [3,4]. Several studies advise starting an anti-tuberculosis drug treatment at an early stage as surgical treatment is not always the method of choice [1-6].

In the present study, mycobacterial cultures from samples provided by aspiration during ultrasonography and from surgical evacuation of the abscess were negative. However, polymerase chain reaction was performed and M. tuberculosis complex was detected. According to studies by Hengster et al. [5] and Aribas et al. [7], bacteria can be cultured from approximately $46 \%$ of individuals within 20 weeks of vaccination but may not be cultured afterwards. Bacteria could not be cultured in this study as too much time had passed since the vaccination. In patients without cultured bacteria or any history of contact with patients with tuberculosis, it is not easy to suspect the cause of an abscess would be tuberculosis. Based on the polymerase chain reaction results indicating detection of $M$. tuberculosis complex from the preoperative ultrasound aspiration sample, as well as the sample biopsy results obtained during the surgery and the radiological examination results, we made a definitive diagnosis of tuberculosis infection and therefore an anti-tuberculosis drug treatment was administered.

The regimen for anti-tuberculosis drug treatment may differ depending on the country of treatment or the therapist $[3,4]$. In several papers, a regimen consisting of isoniazid and rifampicin were used in children [3], and the World Health Organization's 2014 guideline recommends administering isoniazid (10 mg/kg, range of $10-20 \mathrm{mg} / \mathrm{kg}$, maximum $300 \mathrm{mg} /$ day) and rifampicin $(15 \mathrm{mg} / \mathrm{kg}$, range of $7-15 \mathrm{mg} / \mathrm{kg}$, maximum 600 $\mathrm{mg}$ /day) for more than 6 months as first-line anti-tuberculosis treatment for children with tuberculosis.

Several case studies, though with symptoms different from those of the infant in our study, reported the presence of abscess after administration of the BCG vaccine in patients aged 8 to 17 months. Even if the small number of cases prevents robust statistical analysis, it should be acknowledged that complications can occur several months after administration of the BCG vaccine. The possibility of "BCG-oma" should be considered when diagnosing lesions on children aged 12 months old $[3,7,8]$.

Masses that develop in the chest wall of children can be due to an underlying disease, congenital abnormality, trauma, 
neoplasm, or infection [9]. Differential diagnosis is important for these masses on the chest because treatment is determined by the cause. Therefore, it is important to have precise clinical records on the birth, past, and contact histories and to accurately assess the condition of the patient at the time of visit by conducting laboratory tests. Most importantly the mass must be differentiated from other granulomatous masses through a histopathological test that confirms it is a BCG vaccinationinduced infection. To conclude, as the mass had developed in the infant of the present study due to administration of the BCG vaccine, a detailed medical history, physical examination, radiology, and biopsy would be useful to establish a positive diagnosis in patients with similar clinical signs and epidemiological context.

\section{Conflict of interest}

This work was supported by Chosun University Research Fund. Otherwise, no potential conflicts of interest relevant to this article are reported.

\section{ORCID iDs}

Dong Seok Shin https://orcid.org/0000-0001-7062-2815

Min Hyub Choi https://orcid.org/0000-0002-6844-0526

Woo Young Choi https://orcid.org/0000-0001-8849-1569

Ji Seon Cheon https://orcid.org/0000-0001-8555-5088

Kyung Min Son https://orcid.org/0000-0001-5825-0270

\section{References}

1. Talbot EA, Perkins MD, Silva SF, et al. Disseminated bacille Calmette-Guérin disease after vaccination: case report and review. Clin Infect Dis 1997;24:1139-46.

2. Lee HS, Seo KJ, Kim JJ. Chest wall granuloma associated with BCG vaccination presenting as hot abscess in an immunocompetent infant. J Cardiothorac Surg 2015;10:29.

3. Choi YY, Han MS, Lee HJ, et al. Mycobacterium bovis osteitis following immunization with bacille CalmetteGuérin (BCG) in Korea. J Korean Med Sci 2018;34:e3.

4. Lin WL, Chiu NC, Lee PH, et al. Management of bacillus Calmette-Guérin osteomyelitis/osteitis in immunocompetent children: a systematic review. Vaccine 2015;33:4391-7.

5. Hengster P, Solder B, Fille M, et al. Surgical treatment of bacillus Calmette Guérin lymphadenitis. World J Surg 1997; 21:520-3.

6. Huang CY, Su WJ, Perng RP. Childhood tuberculosis presenting as an anterior chest wall abscess. J Formos Med Assoc 2001;100:829-31.

7. Aribas OK, Kanat F, Gormus N, et al. Cold abscess of the chest wall as an unusual complication of BCG vaccination. Eur J Cardiothorac Surg 2002;21:352-4.

8. Kim DH, Choi CW. Chest wall abscess likely due to BCG vaccination in a child. Infection 2009;37:286-8.

9. Baez JC, Lee EY, Restrepo R, et al. Chest wall lesions in children. AJR Am J Roentgenol 2013;200:W402-19. 